\title{
INSTABILITÉ CHROMOSOMIQUE RADIOINDUITE DANS LES FIBROBLASTES HUMAINS
}

\author{
Laure SABATIER, Berta MARTINS, Michelle RICOUL et \\ Bernard DUTRILLAUX
}

Commissariat à l'Energie Atomique, DSV/DPTE/LCG

BP. 6, 92265 Fontenay-aux-Roses

Institut Curie, URA 620 CNRS,

26 rue d'Ulm, 75005 Paris cedex

Il n'existe pour l'instant que peu de données sur les effets biologiques des ions lourds (Tobias, 1985; Lett et al, 1986, 1992). Il a récemment été démontré que les particules à transfert linéique d'énergie (TLE) élevé induisaient une mutagénèse chromosomique particulière. Les lésions chromosomiques sont plus complexes que celles induites par les rayons $\mathrm{X}$ ou $\gamma$, elles impliquent plusieurs chromosomes et leur complexité s'accroît lorsque le TLE des particules augmente (Sabatier et al., 1987; 1990). La fréquence des mitoses présentant de tels remaniements chromosomiques chute de $30 \%$ à chaque génération cellulaire suivant l'irradiation (Al Achkar et al, 1988). Ainsi, après quelques générations, presque toutes les cellules porteuses de remaniements chromosomiques complexes devraient disparaître. De plus, les particules lourdes induisent très efficacement la mort cellulaire (Kraft et al, 1987, Kiefer, 1989). Cependant, quelques cellules porteuses de remaniements chromosomiques radioinduits leur conférant un avantage prolifératif pourraient survivre et constituer des clones envahissant les cultures. Cette hypothèse est confortée par la grande efficacité de ces particules dans l'induction de la transformation cellulaire (Yang et al, 1985, Suzuki et al, 1990).Les liens entre l'observation de remaniements chromosomiques et l'apparition d'une transformation cellulaire radioinduite ne sont pas connus.

Récemment, Kadhim et al (1992) ont montré, après irradiation de cellules hématopoïétiques de souris par des particules $\alpha$ du Pu-238 (TLE $=120 \mathrm{keV} / \mu \mathrm{m}$ ), l'apparition d'une instabilité chromosomique. Nos travaux, sur des cultures primaires de fibroblastes humains irradiés par des ions lourds couvrant une large gamme de TLE (386-13600 keV/um), ont aussi permis de démontrer qu'une instabilité chromosomique était acquise de novo dans les cellules humaines et que les lésions chromosomiques n'étaient pas aléatoires (Sabatier et al 1992).

L'analyse des 7 cultures irradiés au GSI (Darmstadt) par des ions Neon ( $E=10,74 \mathrm{MeV} / \mathrm{u}$, $T L E=386 \mathrm{keV} / \mu \mathrm{m}$; fluences : $10^{6}, 2.10^{6}$ et $4.10^{6}$ particules $\left./ \mathrm{cm}^{2}\right)$, des ions Argon $(\mathrm{E}=10,52$ $\mathrm{MeV} / \mathrm{u}, \mathrm{TLE}=1207 \mathrm{keV} / \mu \mathrm{m}$; fluences : $10^{6}, 2.10^{6}$ et $4.10^{6}$ particules $/ \mathrm{cm}^{2}$ ) et des ions Plomb $\left(\mathrm{E}=9,5 \mathrm{MeV} / \mathrm{u}, \mathrm{TLE}=1360 \mathrm{keV} / \mu \mathrm{m}\right.$; fluence $: 2.10^{6}$ particules $\left./ \mathrm{cm}^{2}\right)$ a donné des résultats similaires pour toutes les cultures cellulaires et nous a permis de proposer le schéma suivant : 
- L'irradiation par des ions lourds induit des aberrations chromosomiques multiples et complexes.

- La plupart des cellules traversées par une particule (ou plus) meurent ou n'engendrent pas de descendants viables. De ce fait, la majorité des métaphases présente un caryotype normal vers les passages 5-10 in vitro après irradiation.

- A partir du 15ième passage, une instabilité chromosomique apparaît.

- Cette instabilité affecte spécifiquement les régions télomériques des chromosomes 13,16 et du bras court du chromosome 1.

- Certains clones, caractérisés par des remaniements et des déséquilibres chromosomiques (en particulier, monosomie 13) envahissent progressivement certaines cultures.

Ces résultats suggèrent que les cellules survivantes ont acquis, après une période d'instabilité chromosomique, des anomalies retrouvées dans des cellules transformées ou tumorales.

L'apparition de novo d'une instabilité chromosomique radioinduite n'a jusqu'ici pas été observée après irradiations à faible TLE. Cependant l'instabilité chromosomique n'est pas un phénomène induit par les seuls ions lourds. En effet, elle est généralement observée au cours de la sénescence et dans des pathologies prémalignes ou de bas grade de malignité (Benn, 1976; Kovacs et al., 1988; Mandhal et al., 1985; Pathak et al., 1988; Aledo et al., 1988). L'instabilité chromosomique pourrait être l'un des mécanismes à l'origine du passage d'une cellule diploide normale à une cellule aneuploïde transformée. Après irradiation par des ions lourds, les fibroblastes survivants auraient donc franchi une étape de la transformation cellulaire.

\section{Références}

Al Achkar, W., L. Sabatier and B. Dutrillaux (1988) Mutation Res., 198, 191-198.

Aledo R., M. F. Avril, B. Dutrillaux and A. Aurias (1988) Cancer Genet Cytogenet., 33, $29-33$. Benn P.B. (1976) Am.J.Hum.Genet., 28, 465-473.

Fry R.J.M., P. Powewers-Risius, E.L. Alpen and E.J. Ainsworth (1985) Radiation Res., Suppl. 8, 104, S-188-S-195.

Kadhim M.A., D.A. Macdonald, D.T. Goodhead, S.A. Lorimore, S.J. Mardsen and E.G. Wright (1992) Nature., 355, 738-740.

Kiefer J. (1989) In Biological Radiation Action Ed Springer-Verlag.

Kovacs G., R. Muller-Brechlin and Szucs S (1987) Cancer Genet Cytogenet., 32, 93-101.

Lett J.T., A.B. Cox and D.S. Bergtold (1986) Radiat. Environ. Biophys., 25, 1-12.

Lett J.T. (1992) Radiat. Environ. Biophys., (in press).

Mandahl N., S.Heim, V. Kristofferson, F. Mitelman, B.Rooser, A.Rydholm and H.Willen (1985) Hum Genet., 71,321-324.

Patak S., Z.Wang, M.K. Dhaliwal and Saks P.C (1988) Cytogenet Cell Genet., 47, 227-229.

Sabatier L. W. Al Achkar and B. Dutrillaux (1990) In frontiers in radiation Biology Ed. E. Riklis, VCH., 287-294.

Sabatier L., W. Al Achkar, F. Hoffschir, C. Luccioni and B. Dutrillaux (1987) Mutation Res., 178,91-97.

Sabatier L., B. Dutrillaux and B. Martins (1992) Nature (London), 357, 548.

Suzuki M., M. Watanabe, K. Suzuki, K. Nakano and I. Kaneko (1989) Radiation Res., 120, 468-476.

Tobias C. A. (1985) Radiation Research., 103, 1-33.

Yang T. C., L.M. Craise, M.T. Mei and C.A. Tobias (1985) Radiation Res., 104, S-177-S-187. 